ISSN: $2354-1431$

http://tckh.daihoctantrao.edu.vn/

\title{
Nghiên cứu mối quan hệ giữa chất lượng dịch vụ đào tạo với sự hài lòng của sinh viên trường Đại học Tân Trào
}

\author{
Nguyễn Khải Hoàn ${ }^{a, *}$ Nguyễn Phương Thảo ${ }^{a,}$ \\ a Truờng Đại học Tân Trào \\ *Email:hoannk63@gmail.com
}

\section{Article info}

Recieved:

05/7/2017

Accepted:

03/8/2017

\section{Keywords:}

Quality of services;

Student's satisfaction;

Tan Trao university.

\begin{abstract}
This article presents studied results of the connection between quality of training services and student's satisfaction at TTU. The result based on questionnaires from 396 students with 26 questions regarding 5 factors affecting quality of training services (reliability, applicable potential, service capacity, sympathy, facilities) and student's satisfaction. The study shows that sympathy and service capacity most impact on student's satisfaction.
\end{abstract}

\section{Bối cảnh và vấn đề}

Trong quản lý chất lượng hiện đại, triết lý hướng đến khách hàng đang đóng vai trò chủ đạo. Một trong những yếu tố quyết định cho sự tồn tại và phát triển của các đơn vị kinh doanh nói chung và các đơn vị trong lĩnh vực giáo dục nói riêng là sự hài lòng của khách hàng về chất lượng dịch vụ - sản phẩm mà đơn vị cung ứng. Chất lượng phải được đánh giá bởi chính những khách hàng đang sử dụng chứ không phải bởi các đơn vị. Như vậy, trong lĩnh vực giáo dục việc đánh giá chất lượng dịch vụ qua ý kiến của khách hàng, trong đó khách hàng trọng tâm - người học (sinh viên) đang trở lên hết sức cần thiết. Qua đó, các đơn vị đào tạo nói chung và các trường đại học nói riêng có các nhìn nhận khách quan về những gì mình đã cung cấp, những gì mình kỳ vọng thay vì chỉ quan tâm đến đầu tư cơ sở vật chất, trình độ đầu vào - đầu ra và kết quả học tập của sinh viên và các yếu tố khác trong quá trình đào tạo.

Xét trên góc độ kinh tế học giáo dục thì giáo dục đại học hiện nay được coi là một loại hình dịch vụ quan trọng trong việc cung cấp dịch vụ đào tạo để thu hút sinh viên đến với trường của mình. Chính vì vậy, việc nâng cao chất lượng dịch vụ đào tạo là một quá trình cần được thực hiện liên tục trong chiến lược phát triển của mỗi trường đại học. Thông qua những ý kiến nhìn nhận về chất lượng dịch vụ đào tạo từ phía sinh viên, những người đang trực tiếp hưởng dịch vụ đào tạo là một thành phần đóng góp không thể thiếu trong việc thực hiện cải tiến và nâng cao chất lượng đào tạo của các trường đại học, đặc biệt là các trường đại học địa phương trong xu thế hội nhập, toàn cầu hóa hiện nay.

Mục đích chính của nghiên cứu này là xác định các thành phần tác động vào sự hài lòng của sinh viên về chất lượng dịch vụ đào tạo của Trường Đại học Tân Trào; xây dựng và đánh giá các thang đo lường các thành phần. Để khẳng định sự tác động của các thành phần này vào sự hài lòng của sinh viên, một mô hình lý thuyết được xây dựng và kiểm định. Mô hình lý thuyết được xây dựng dựa trên cơ sở lý thuyết về chất lượng dịch vụ, sự hài lòng của sinh viên và các thành phần tác động vào sự hài lòng.

\section{Nội dung nghiên cứu}

\subsection{Mô hình và giả thuyết nghiên cứu}

Mô hình của Parasuraman \& ctg (1988)[10] đã mô tả khái quát về chất lượng dịch vụ, các nhà nghiên cứu này cũng đã đưa ra được thang đo SERVQUAL để đo lường chất lượng dịch vụ gồm 10 thành phần; thang đo này được Cronin \& Taylor (1992)[7] thay thế bằng thang đo SERVPERF. Thang đo SERVPERF khẳng định "chất lượng dịch vụ = mức độ cảm nhận” và khắc phục những hạn chế của thang đo SERVQUAL. bằng mô hình rút gọn 
gồm 5 thành phần: (i) Tin cậy; (ii) Đáp ứng; (iii) Bảo đảm; (iv) Đồng cảm; (v) Phương tiện hữu hình.

Áp dụng thang đo SERVPERF, Nguyễn Thị Xuân Hương và các cộng sự (2013)[3] đã đưa ra mô hình nghiên cứu đề xuất nghiên cứu sự hài lòng của sinh viên với điều kiện cơ sở vật chất và phục vụ của Trường Đại học Lâm nghiệp gồm 5 thành phần: (i) Cơ sở vật chất; (ii) Năng lực phục vụ; (iii) Sự quan tâm; (iv) Khả năng đáp ứng; (v) Mức độ tin cậy.

Khi nghiên cứu về đánh giá sự hài lòng của học sinh - sinh viên về chất lượng chương trình đào tạo tại Trường Cao đẳng kinh tế đối ngoại, Phan Thị Thanh Hằng (2014)[2] đã đưa ra mô hình nghiên cứu gồm 4 thành phần: (i) Chương trình đào tạo; (ii) Đội ngũ giảng viên; (iii) Cơ sở vật chất; (iv) Khả năng phục vụ̣.

Từ những cơ sở lý thuyết và thực tiễn nêu trên, chúng tôi đưa ra mô hình nghiên cứu đề xuất (Hình 1) để đo lường sự hài lòng của sinh viên về chất lượng dịch vụ đào tạo tại Trường Đại học Tân Trào thông qua mối quan hệ giữa các thành phần chất lượng dịch vụ và sự hài lòng của khách hàng.

Hình 1. Mô hình nghiên cưu sụ hài lòng của sinh viên đối vói chất luợng dịch vu tại truoòng Đại học Tân Trào

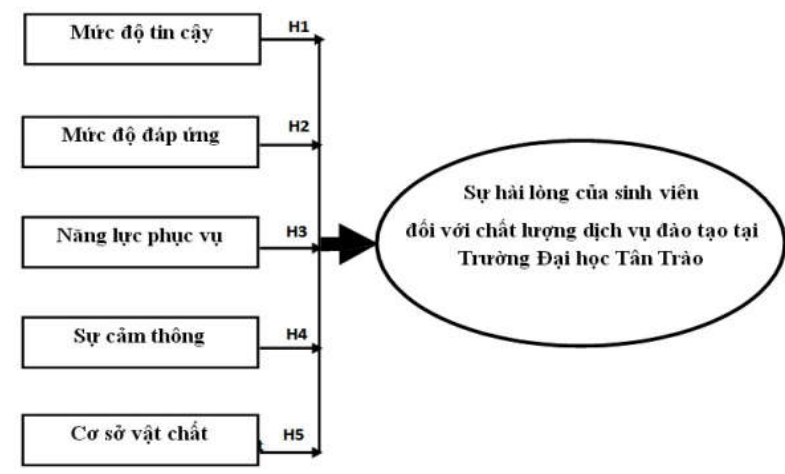

Từ mô hình nghiên cứu đề xuất, các giả thuyết của nghiên cứu được xác định như sau:

H1: Khi mức độ tin cậy được sinh viên đánh giá cao hoặc thấp thì mức độ hài lòng của sinh viên sẽ cao hoặc thấp tương ứng.

H2: Khi mức độ đáp ứng được sinh viên đánh giá cao hoặc thấp thì mức độ hài lòng của sinh viên sẽ cao hoặc thấp tương ứng.

H3: Khi năng lực phục vụ được sinh viên đánh giá cao hoặc thấp thì mức độ hài lòng của sinh viên sẽ cao hoặc thấp tương ứng.

H4: Khi sự cảm thông được sinh viên đánh giá cao hoặc thấp thì mức độ hài lòng của sinh viên sẽ cao hoặc thấp tương ứng.
H5: Khi cơ sở vật chất được sinh viên đánh giá cao hoặc thấp thì mức độ hài lòng của sinh viên sẽ cao hoặc thấp tương ứng.

\subsection{Dữ liệu và phương pháp nghiên cứu}

Nghiên cứu được thực hiện qua hai bước: (i) Nghiên cứu sơ bộ sử dụng phương pháp định tính được tiến hành thông qua kỹ thuật thảo luận nhóm, phỏng vấn nhằm phát hiện, điều chỉnh và bổ sung các biến quan sát dùng để thực hiện đo lường các khái niệm nghiên cứu; (ii) Nghiên cứu chính thức sử dụng phương pháp định lượng được thực hiện thông qua hình thức phỏng vấn trực tiếp thông qua bảng câu hỏi chi tiết mục đích là đánh giá các thang đo và kiểm định mô hình lý thuyết của đề tài.

Trong quá trình nghiên cứu sơ bộ, thảo luận nhóm được tiến hành với 18 cán bộ, giảng viên và 15 sinh viên nhằm hiệu chỉnh thang đo sao cho dễ hiểu và phù hợp với đặc thù của một trường Đại học địa phương. Nghiên cứu chính thức là giai đoạn thu thập dữ liệu bằng bảng câu hỏi với 26 biến quan sát phản ánh 5 yếu tố ảnh hưởng tới chất lượng dịch vụ đào tạo, 6 biến quan sát phản ánh sự hài lòng của sinh viên và sử dụng thang điểm Likert đánh từ $1-5$ điểm. Quy mô khảo sát thực tế là 415 mẫu được thực hiện trong năm 2017. Cả 2 nghiên cứu trên đều được thực hiện tại Trường Đại học Tân Trào với đối tượng nghiên cứu là sinh viên hệ đại học, cao đẳng chính qui đang học tập ở các khoa: Giáo dục tiểu học; Giáo dục mầm non; Khoa học Xã hội và Nhân văn; Khoa học Tự nhiên - Kỹ thuật - Công nghệ; Nông - Lâm - Ngư nghiệp; Kinh tế và Quản trị kinh doanh. Sau khi được làm sạch loại bỏ các bảng không có giá trị thì mẫu chính thức là $\mathrm{n}=396$. Kết quả chính thức được sử dụng để phân tích, đánh giá thang đo lường các thành phần tác động vào sự hài lòng của sinh viên thông qua hệ số tin cậy Cronbach Alpha, phân tích nhân tố EFA, kiểm định mô hình lý thuyết và các giả thuyết thông qua phân tích phương sai một nhân tố ANOVA.

\subsection{Kết quả nghiên cứu}

\subsubsection{Phân tích chung về số liệu khảo sát sinh viên}

Theo Bảng 1, số sinh viên được nghiên cứu tập trung tại 6 khoa với số lượng sinh viên: nữ là 257 , chiếm $64,9 \%$, nam là 157 , chiếm $35,1 \%$. Số liệu trên phản ánh đúng thực tế tỷ lệ sinh viên nam và nữ trong Trường Đại học Tân Trào. 
Bảng 1: Thông tin chung về số liệu khảo sát

\begin{tabular}{|c|c|c|c|c|}
\hline Giới tính & Frequency & Percent & Valid Percent & $\begin{array}{l}\text { Cumulativ } \\
\text { e Percent }\end{array}$ \\
\hline Nam & 139 & 35.1 & 35.1 & 35.1 \\
\hline Nữ & 257 & 64.9 & 64.9 & 100.0 \\
\hline Tổng cộng & 396 & 100.0 & 100.0 & \\
\hline \multicolumn{5}{|l|}{ Khoa } \\
\hline GDTH & 118 & 29.8 & 29.8 & 29.8 \\
\hline GDMN & 79 & 19.9 & 19.9 & 49.7 \\
\hline KHXH-NV & 50 & 12.6 & 12.6 & 62.4 \\
\hline KHTN-KT-CN & 50 & 12.6 & 12.6 & 75.0 \\
\hline NL-NN & 49 & 12.4 & 12.4 & 87.4 \\
\hline KT\&QTKD & 50 & 12.6 & 12.6 & 100.0 \\
\hline Tổng cộng & 396 & 100.0 & 100.0 & \\
\hline
\end{tabular}

( Nguồn: Tổng hợp nghiên cúu tại Trưòng Đại học Tân Trào tháng 02/2017)

\subsubsection{Phân tích thang đo}

a) Đánh giá độ tin cậy của thang đo qua hệ số tin cậy Cronbach Alpha

Kết quả phân tích Cronbach Alpha cho ta thấy 5 thành phần của thang đo chất lượng dịch vụ đào tạo thông qua ý kiến đánh giá của sinh viên đều có độ tin cậy lớn hơn 0,6 . Như vậy, thang đo thiết kế trong bài viết có ý nghĩa trong thống kê và đạt hệ số tin cậy cần thiết.

Bảng 2: Kết quả đánh giá độ tin cậy của thang đo với các nhân tố sau:

\begin{tabular}{|c|c|c|c|c|c|}
\hline Nhân tố & $\begin{array}{c}\text { Độ tin } \\
\text { cậy }\end{array}$ & $\begin{array}{c}\text { Mức độ } \\
\text { đáp ứng }\end{array}$ & $\begin{array}{c}\text { Năng lực } \\
\text { phục vụ }\end{array}$ & $\begin{array}{c}\text { Sự cảm } \\
\text { thông }\end{array}$ & $\begin{array}{c}\text { Cơ sở } \\
\text { vật chất }\end{array}$ \\
\hline $\begin{array}{c}\text { Hệ số } \\
\text { Cronbach } \\
\text { Alpha }\end{array}$ & 0,746 & 0,860 & 0,829 & 0,810 & 0,882 \\
\hline
\end{tabular}

(Nguồn: Kết quả xủ lý số liệu trên phần mềm SPSS)

b) Phân tích nhân tố khám phá EFA

Theo mô hình nghiên cứu có 5 nhân tố với 26 biến quan sát ảnh hưởng đến ý kiến đánh giá của $\mathrm{SV}$ đối với chất lượng đào tạo của nhà trường. Sau khi khảo sát, dùng phương pháp phân tích nhân tố khám phá EFA với phép quay Varimax để phân tích 26 biến quan sát. Sử dụng phương pháp kiểm định KMO (Kaiser-MeyerOlkin) và Bartlett để đo lường sự tương thích của mẫu khảo sát. Kết quả được thể hiện ở Bảng 3 sau đây:
Bảng 3: Kiểm định KMO và Bartlett's Test

\begin{tabular}{|c|c|c|}
\hline \multicolumn{2}{|c|}{ Kaiser-Meyer-Olkin Measure of Sampling Adequacy. } & 0.908 \\
\hline \multirow[t]{3}{*}{ Bartlett's Test of Sphericity } & Approx. Chi-Square & 5378.448 \\
\hline & df & 325 \\
\hline & Sig. & 0.000 \\
\hline
\end{tabular}

(Nguồn: Kết quả xủ lý số liệu trên phần mềm SPSS)

Với giả thiết $\mathrm{H}_{0}$ đặt ra trong phân tích này là giữa 26 biến quan sát trong tổng thể không có mối tương quan với nhau. Hệ số KMO là $\mathbf{0 , 9 0 8}(>0,5)$ và $\operatorname{sig}=\mathbf{0 , 0 0 0}<\mathbf{0 , 0 5}$ điều này cho thấy giả thiết $\mathrm{H}_{0}$ bị bác bỏ $(\mathrm{sig} .=0,000)$, do vậy các biến quan sát có tương quan với nhau trong tổng thể và phân tích nhân tố EFA là thích hợp. Trong 26 biến quan sát không có giá trị nào không đạt yêu cầu (các trọng số nhân tố đều lớn hơn 0,4 ; thấp nhất là 0,539 và cao nhất là 0,888$)$, bên cạnh đó các biến quan sát không tách thành những nhóm nhân tố mới đồng nghĩa với thang đo đạt tiêu chuẩn, 5 nhân tố được rút trích và có thể sử dụng chúng trong việc phân tích tiếp theo để thu được kết quả nghiên cứu mong muốn.

\subsubsection{Phân tích hồi quy}

\section{a) Mô tả các nhân tố đo lường mức độ hài lòng}

Theo ý kiến đánh giá của sinh viên thì các yếu tố ảnh hưởng trực tiếp tới chất lượng đào tạo của nhà trường bao gồm 5 nhân tố sau: NLPV (mean $=3.33)$ có nghĩa sinh viên đánh giá ở mức trung bình, không có ý kiến gì với nhân tố này; 4 nhân tố: CSVC $($ mean $=3,48)$, SCT (mean 
$=3,64)$; DU $($ mean $=3.69) ;$ TC $($ mean $=3,80)$ cho thấy đã phần lớn làm sinh viên hài lòng với chất lượng dịch vụ đào tạo tại trường. Tuy nhiên, để biết được trong các nhân trên nhân tố nào ảnh hưởng nhiều, nhân tố nào ảnh hưởng ít đến sự hài lòng của sinh viên cần tiếp tục phân tích tương quan, hồi quy để xác định những nhân tố ảnh hưởng này.

\section{b) Phân tích tuoong quan Pearson}

Phân tích tương quan hệ số Pearson là để lượng hóa mức độ chặt chẽ của mối liên hệ tuyến tính giữa các biến định lượng. Kết quả cho thấy các hệ số tương quan đều lớn ở mức hơn 0.6, thể hiện mối liên hệ tương quan tuyến tính giữa các biến khá chặt chẽ, cao nhất $=0.754$, thấp nhất $=0.602$ không vượt quá hệ số điều kiện 0,85 ( John \& Bener - Martinez, 2000). Điều đó chứng minh giá trị phân biệt giữa các nhân tố đã đạt được.

\section{c) Phân tích hồi quy bội và kiểm đinh giả thuyết}

Bảng 4: Kết quả hồi quy

\begin{tabular}{|c|c|c|c|}
\hline Nhân tố & Beta & $\begin{array}{c}\text { Giá trị } \\
\text { Sig }\end{array}$ & $\begin{array}{c}\text { Hệ số phóng đại } \\
\text { phương sai }\end{array}$ \\
\hline TC & .194 & .000 & 1.447 \\
\hline DU & .173 & .000 & 1.813 \\
\hline NLPV & .318 & .000 & 2.174 \\
\hline SCT & .357 & .000 & 1.957 \\
\hline CSVC & -.002 & .000 & 1.074 \\
\hline $\begin{array}{c}\mathrm{R}^{2}=0.737 \\
\text { F=218,393 } \\
\text { (Sig.F }=0.000)\end{array}$ & & & \\
\hline
\end{tabular}

(Nguồn: Kết quả xủ lý số liệu trên phần mềm SPSS)

Giá trị $\mathrm{R}$ bình phương $=0.737$ tức $73,7 \%$ sự biến thiên của biến Sự hài lòng được giải thích bởi 5 thành phần trong chất lượng dịch vụ đào tạo. Do vậy, mức độ phù hợp mô hình khá tốt. Mặt khác để kiểm định sự phù hợp của mô hình với áp dụng thực tế ta có thể thấy giá trị Sig. của trị $\mathrm{F}$ của mô hình rất nhỏ ( $<$ mức ý nghĩa: 0,05 ) =>bác bỏ giả thiết $\mathrm{Ho}=>$ mô hình phù hợp với tập dữ liệu và có thể suy rộng ra cho toàn tổng thế. Hệ số phóng đại phương sai rất nhỏ (không vượt quá 10) cho thấy sự đa cộng tuyến rất thấp, xác nhận rằng thang đo lường không bị trùng lặp,

Mặt khác các yếu tố TC, DU, NLPV, SCT đều có hệ số Beta dương và mức ý nghĩa Sig. $=0,000<0.05$ nên các biến độc lập này đều có tác động thuận chiều đến Sự hài lòng của sinh viên hay nói cách khác: khi cảm nhận của sinh viên về chất lượng dịch vụ đào tạo tăng lên thì sự hài lòng của sinh viên cũng tăng theo. Do vậy, giả thuyết $\mathrm{H} 1$, H2, H3, H4, H5 đều được chấp nhận. Riêng yếu tố CSVC có giá trị Beta âm rất nhỏ và mức ý nghĩa Sig. $>0,05$ nên không có ý nghĩa thông kê hay xét về mặt nội dung thì biến độc lập CSVC không có tác động đến sự hài lòng của sinh viên Trường Đại học Tân Trào, do vậy giả thuyết H5 bị loại khỏi mô hình.

Mức độ ảnh hưởng của các nhân tố thể hiện qua phương trình hồi quy bội là:

\section{$\mathrm{SHL}=0.194^{\star} \mathrm{TC}+0.173^{\star} \mathrm{DU}+0.318^{\star} \mathrm{NLPV}+0.357^{\star} \mathrm{SCT}$}

Phương trình trên cũng cho thấy rằng trong các yếu tố tác động và ảnh hưởng đến sự hài lòng của sinh viên thì hai biến "Sự cảm thông" và "Năng lực phục vụ" đều có giá trị lớn nhất tức hai biến này ảnh hưởng nhiều nhất đến sự hài lòng của sinh viên về chất lượng dịch vụ đào tạo của Trường Đại học Tân Trào. Tiếp đến là yếu tố “ Mức độ tin cậy” và “ Mức độ đáp ứng”.

\section{Kết luận}

Kết quả nghiên cứu cho thấy 4 nhân tố ảnh hưởng đến chất lượng dịch vụ đào tạo có tác động cùng chiều lên sự hài lòng của sinh viên: Năng lực phục vụ, Sự cảm thông, Mức độ đáp ứng, Độ tin cậy. Đồng thời nghiên cứu chỉ ra mức độ khác nhau giữ những nhân tố trên, mức độ tác động nhiều hơn cả là: Sự cảm thông và Năng lực phục vụ. Đối với yếu tố Cơ sở vật chất không được thừa nhận là biến dự báo về sự hài lòng của sinh viên trong nghiên cứu và đây cũng là yếu tố có ảnh hưởng thấp nhất lên sự hài lòng, tuy nhiên không có ý nghĩa trong dự báo do kiểm định không có ý nghĩa thống kê.

Trong nghiên cứu này, các yếu tố định tính đã được định lượng hóa một cách khoa học, phù hợp và dễ dàng. Tuy nhiên, để làm rõ được những nguyên nhân, tồn tại, khắc phục những hạn chế về chất lượng dịch vụ đào tạo của nhà trường, phục vụ tốt hơn nhu cầu của người học cần mở rộng đối tượng khảo sát, quan tâm đến những nhân tố khác: nhân tố tình huống, kết quả đầu vào, kết quả đầu ra...trong các nghiên cứu tiếp theo để có thể đưa ra được những giải pháp hữu hiệu đáp ứng yêu cầu quản trị đại học trong giai đoạn hiện nay.

\section{TÀI LIỆU THAM KHẢO}

1. Nguyễn Thị Bảo Châu, Thái Thị Bích Châu (2013). Đánh giá mức độ hài lòng của sinh viên đối với chất luợng đào tạo của Khoa Kinh tế và Quản trị kinh doanh Trường Đại học Cần Tho giai đoạn năm 2012 -2013. Tạp chí khoa học Trường Đại học Cần Thơ, số 28(2013), tr.117-123;

2. Phan Thị Thanh Hằng (2014), Sụ hài lòng của hoc sinh - sinh viên về chất luợng chuoong trình đào tạo tại truoòng Cao đẳng kinh tế đối ngoại. Chuyên san Kinh tế đối ngoại, kỳ 11, tr.13-20; 
3. Nguyễn Thị Xuân Hương, Nguyễn Thị Phượng, Vũ Thị Hồng Loan (2016). Các nhân tố ảnh hưởng đến sự hài lòng của sinh viên với điều kiện cơ sở vật chất và phuc vu của Truờng Đại học Lâm nghiệp. Tạp chí Khoa học và công nghệ lâm nghiệp, số 2-2016;

4. Hoàng Trọng, Chu Nguyễn Mộng Ngọc (2005), Phân tích dĩ liệu nghiên cưu với SPSS, Nxb Thống kê;

5. Nguyễn Đình Thọ (2012), Phuơng pháp nghiên cưu khoa học trong kinh doanh. Nxb Lao động, Tp. HCM;

6. Lại Xuân Thủy, Phan Thị Minh Lý (2011), Đánh giá chất luợng đào tạo tại khoa Kế toán - Tài chính, Truờng Đại học Kinh tế, Đại học Huế trên quan điểm của nguời học, Tạp chí khoa học và công nghệ, Đại học Đà Nã̃ng, số $3(44)$;

7. Cronin, J.J \& S.A. Taylor (1992), Measuring Service
Quality: A Reexamination and Extension, Journal of Marketing, 56 (July): 55-68;

8. Juran, J.M. (1988), Juran's Quality Control Handbook. Four Edition, New York: McGraw-Hill;

9. John, O.P. \& Benet-Martínez, V. (2000). Measurement, scale construction, and reliability. In H.T. Reis and C.M. Judd (Eds.), Handbook of research methods in social and personality psychology (pp. 339-369). New York, NY: Cambridge University Press;

10. Parasuraman, A., V.A. Zeithaml, \& L. L. Berry (1988), SERVQUAL: a mutltiple-item scale for measuring consumer perceptions of service quality, Journal of Retailing, 64(1): 12-40;

11. Parasuraman, A., L. L. Berry, \& V. A. Zeithaml (1991), Refinement and Reassessment of the SERVQUAL Scale, Journal of Retailing, 67 (4): 420-450. 